\title{
In vivo proton magnetic resonance spectroscopy (1H-MRS) evaluation of the metabolite concentration of optic radiation in primary open angle glaucoma
}

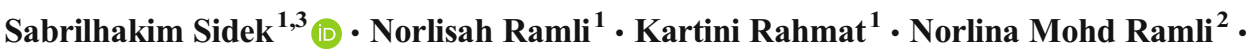 \\ Fadzlina Abdulrahman ${ }^{2}$. Tan Li Kuo ${ }^{1}$
}

Received: 1 July 2015 /Revised: 4 February 2016 / Accepted: 8 February 2016/Published online: 4 March 2016

(C) The Author(s) 2016. This article is published with open access at Springerlink.com

\begin{abstract}
Objective To compare the metabolite concentration of optic radiation in glaucoma patients with that of healthy subjects using Proton Magnetic Resonance Spectroscopy (1H-MRS). Methods 1H-MRS utilising the Single-Voxel Spectroscopy (SVS) technique was performed using a 3.0Tesla MRI on 45 optic radiations ( 15 from healthy subjects, 15 from mild glaucoma patients, and 15 from severe glaucoma patients). A standardised Volume of Interest (VOI) of $20 \times 20 \times 20 \mathrm{~mm}$ was placed in the region of optic radiation. Mild and severe glaucoma patients were categorised based on the HodappParrish-Anderson (HPA) classification. Mean and multiple group comparisons for metabolite concentration and metabolite concentration ratio between glaucoma grades and healthy subjects were obtained using one-way ANOVA.

Results The metabolite concentration and metabolite concentration ratio between the optic radiations of glaucoma patients and healthy subjects did not demonstrate any significant difference $(p>0.05)$.

Conclusion Our findings show no significant alteration of metabolite concentration associated with neurodegeneration that could be measured by single-voxel $1 \mathrm{H}-\mathrm{MRS}$ in optic radiation among glaucoma patients.
\end{abstract}

Sabrilhakim Sidek

bhkimy_7@hotmail.com; sabrilhakim@salam.uitm.edu.my

1 Department of Biomedical Imaging, University Malaya Research Imaging Centre (UMRIC), University of Malaya, Lembah Pantai, 50603 Kuala Lumpur, Malaysia

2 Department of Ophthalmology, Faculty of Medicine, University of Malaya, Lembah Pantai, 50603 Kuala Lumpur, Malaysia

3 Medical Imaging Unit, Faculty of Medicine, Universiti Teknologi MARA, Sg Buloh Campus, 47000 Sg Buloh, Selangor, Malaysia
Key Points

- Glaucoma disease has a neurodegenerative component.

- Metabolite changes have been observed in the neurodegenerative process in the brain.

- Using SVS, no metabolite changes in optic radiation were attributed to glaucoma.

Keywords Glaucoma · Neurodegeneration · Spectroscopy · Magnetic resonance imaging · Metabolites

$\begin{array}{ll}\text { Abbreviations } \\ \text { Ala } & \text { L-alanine } \\ \text { ANOVA } & \text { Analysis of variance } \\ \text { Asp } & \text { Aspartate } \\ \text { Cho } & \text { Choline } \\ \text { CNS } & \text { Central nervous system } \\ \text { Cr } & \text { Creatine } \\ \text { CrCH2 } & \text { Creatine methylene } \\ \text { CRLB } & \text { Cramer-Rao lower bound } \\ \text { DTI } & \text { Diffusion tensor imaging } \\ \text { FOV } & \text { Field of view } \\ \text { FSPGR } & \text { Fast spoiled gradient echo } \\ \text { GCT } & \text { Geniculocalcarine tract } \\ \text { Gln } & \text { Glutamine } \\ \text { Glu } & \text { Glutamate } \\ \text { Glx } & \text { Glu + Gln } \\ \text { GPC } & \text { Glycerophosphocholine } \\ \text { HPA } & \text { Hodapp-Parrish-Anderson } \\ \text { Ins } & \text { Inositol } \\ \text { Lac } & \text { Lactate } \\ \text { LGB } & \text { Lateral geniculate body } \\ \text { Lip } & \text { Lipids } \\ \text { MD } & \text { Mean deviation } \\ \text { MM } & \text { Macromolecules }\end{array}$




$\begin{array}{ll}\text { MRI } & \text { Magnetic resonance imaging } \\ \text { n } & \text { Number of subjects } \\ \text { NAA } & \text { N-acetyl aspartate } \\ \text { NAAG } & \text { N-acetyl aspartylglutamate } \\ \text { NEX } & \text { Number of excitation } \\ \text { ON } & \text { Optic nerve } \\ \text { PCh } & \text { Phosphocholine } \\ \text { PCr } & \text { Phosphocreatine } \\ \text { PRESS } & \text { Point-resolved single-voxel spectroscopy } \\ \text { RNFL } & \text { Retinal nerve fibre layer } \\ \text { SD } & \text { Standard deviation } \\ \text { SPSS } & \text { Statistical Package for the Social Sciences } \\ \text { SVS } & \text { Single-voxel spectroscopy } \\ \text { tCho } & \text { GPC + PCh } \\ \text { tCr } & \text { Cr + PCr } \\ \text { tNAA } & \text { NAA + NAAG } \\ \text { TE } & \text { Echo time } \\ \text { TR } & \text { Repetition time } \\ \text { 1H-MRS } & \text { Proton magnetic resonance spectroscopy } \\ \text { RGC } & \text { Retinal ganglion cell } \\ \text { VBM } & \text { Voxel-based morphometry } \\ \text { VOI } & \text { Volume of interest } \\ \text { \%SD } & \text { Percentage standard deviation for CRLB } \\ & \text { or CRLB percentage value }\end{array}$

\section{Introduction}

Glaucoma is an irreversible neurodegenerative disease and the second most common cause of blindness worldwide [1]. Retinal Ganglion Cell (RGC) death and visual pathway axonal degeneration contribute to glaucoma. The pathophysiology of glaucoma is believed to be multifactorial. Irrespective of the site of neuronal injury, factors, or mechanisms involved, the terminal outcome is the death of RGCs and their axons, thus resulting in irreversible visual loss [2].

Anatomical changes reflecting damage to the optic nerve $(\mathrm{ON})$ head and retinal nerve fibre layer (RNFL) are the basis for glaucoma diagnosis and evaluation. Recent studies have broadened the theory of the neurodegenerative process of glaucoma beyond the $\mathrm{ON}$ per se to involve the entire visual pathway through assessment methods utilising newer and more advanced MRI techniques [3-7].

Previous morphological studies evaluated the volume changes in various specific structures in the brain in patients with glaucoma [8-11]. Using MRI Voxel-Based Morphometry (VBM), Hernowo et al. found significant volume reduction in all structures of the visual pathways, including optic radiations [8]. In the occipital lobe, MRI volumetric studies were specifically focused on the visual and visual association cortices. Recent publications observed significant cortical thinning in the occipital lobe using volumetric MRI techniques or volume reduction of the visual cortices in patients with glaucoma [9-11]. Diffusion Tensor Imaging (DTI) corroborated the findings of decreased microstructural white matter integrity in optic radiations among patients with glaucoma [7, 12]. Interestingly, other MRI volumetric studies conducted by William et al., Li et al. and Chen et al. showed volumetric gains and a decrease in several structural components of the visual cortices in the occipital lobe [13-15].

To the best of our knowledge, only a few studies on the changes in metabolite concentrations in the visual pathway related to glaucoma disease have been previously published, and these studies demonstrated various findings [16-19]. Boucard et al. published the earliest findings on metabolite changes in seven glaucoma and seven age-related macular degeneration patients compared with 12 healthy subjects. They found no significant metabolite changes for NAA/Cr, $\mathrm{Cho} / \mathrm{Cr}$, and $\mathrm{Glx} / \mathrm{Cr}$ in the occipital lobe of each subject using the single-voxel 1H-MRS technique [16].

By contrast, Doganay et al. studied single-voxel 1H-MRS on the LGB and corpus vitreous of 29 glaucoma patients and 13 healthy subjects [17]. They found statistically significant reduction of $\mathrm{Glx} / \mathrm{Cr}$ ratio in both vitreous body and LGB, but no metabolite alteration in $\mathrm{NAA} / \mathrm{Cr}$ and $\mathrm{Cho} / \mathrm{Cr}$ ratios in the LGB. Additionally, they found a lactate peak in the vitreous body of 11 glaucoma patients.

In the most recent study conducted by Yan Zhang et al., changes in metabolite concentration were found in primary glaucoma using multi-voxel 1H-MRS at the geniculocalcarine tract (GCT) and striate areas [18]. They studied 20 glaucoma patients who were age- and gender-matched to 20 healthy volunteers. Significant reductions in $\mathrm{NAA} / \mathrm{Cr}$ and $\mathrm{Cho} / \mathrm{Cr}$ were found in the geniculocalcarine and striate areas of glaucoma subjects compared with healthy subjects [18].

Using an experimental rat model, Kevin et al. conducted a $1 \mathrm{H}-\mathrm{MRS}$ study on glaucoma and found a significant $\mathrm{Cho} / \mathrm{Cr}$ reduction in the left visual cortex side of induced glaucoma as compared with the right side. No significant difference was observed in other metabolites, including NAA and Glx [19].

$1 \mathrm{H}-\mathrm{MRS}$ is a non-invasive technique that enables the detection, identification, and quantification of biochemical compounds or metabolites in the brain tissue. This approach provides physiological and chemical information [20]. Metabolites that can be measured using 1H-MRS include Nacetyl aspartate (NAA), choline (Cho), creatine (Cr), lactate, glutamine, glutamate, lipids, and macromolecules [21]. In their review article, Soares et al. accurately described the functions of each metabolite, as well as the position of the respective metabolite's peak on the spectrum [22]. In addition, Ross et al. described the alteration in metabolite concentration associated with many diseases across the ages. These conditions include brain hypoxia, inborn errors of metabolism, tumours, 
white matter disease, trauma prognosis, and stroke [23]. Other conditions, such as epilepsy, diabetic coma, and hepatic encephalopathy, were also reported to cause changes in metabolite concentration in the brain [24].

In this study, we applied single-voxel 1H-MRS using a $3 \mathrm{~T}$ scanner to the optic radiations to identify any significant metabolite changes between healthy subjects and patients with mild and severe glaucoma.

\section{Materials and methods}

This cross-sectional study was conducted from October 2010 to September 2012. Ethical approval was obtained from the institutional medical review board. Forty-five optic radiations, which demonstrated good, acceptable spectroscopy curves, were selected. Two neuroradiologists performed visual inspection of the individual spectra (NR \& KR). The reliability and quality of the spectroscopy curves were characterised by modelling the normal distribution of the estimated percentage standard deviations (\%SD) on a per-metabolite basis.

The total number of subjects involved in this study was 26 , for a total of 52 optic radiations. Forty-five optic radiations were selected, whereas seven optic radiations were excluded from this study after spectral characterisation and visual review.

All subjects were enrolled from the ophthalmology clinic of a single tertiary teaching hospital. Informed consent was obtained from each subject. The recruited subjects underwent a comprehensive ophthalmological assessment that included a slit lamp examination, standard automated perimetry using the 24-2 program of the Humphrey Visual Field Analyser (Carl Zeiss Meditec, Dublin, CA, USA), and retinal nerve fibre layer thickness using the Cirrus High Definition-Optical Coherence Tomography (HD-OCT) machine (Carl Zeiss Meditec, Dublin, CA, USA). An experienced consultant ophthalmologist (NMR) assessed the glaucoma severity based on the Hodapp-Parrish-Anderson (HPA) classification system
[25]. Briefly, this classification is based on Mean Deviation (MD) scores on Humphrey Visual Field testing. Mild glaucoma refers to those with MD of less than $-6 \mathrm{~dB}$, whereas severe glaucoma refers to those with MD greater than $-12 \mathrm{~dB}$. The inclusion criterion was patients with bilateral symmetrical disease from primary open angle glaucoma. The exclusion criteria were as follows: (1) patients under 18 years of age, (2) with secondary glaucoma; and (3) with history of any central nervous system (CNS) disorder, including small vessel ischaemic white matter disease found on the initial MRI.

The healthy subjects were either relatives accompanying the subjects or those who attended the clinic for non-glaucomarelated problems. Standard routine ophthalmological investigations, which included slit lamp examination, intraocular pressure measurement with the Goldman tonometer, and optic disc assessment, were performed. Those with large cup disc ratios of 0.7 or more or who had any features suggestive of glaucomatous optic neuropathy were excluded. The demographic information is summarised in Table 1. The larger cohort of healthy subjects and glaucoma patients had undergone concurrent MRI volumetric optic nerve measurement and Diffusion Tensor Imaging (DTI) of the optic nerve and optic radiation, the results of which had recently been published $[6,7]$.

\section{MRI acquisition}

All participants were scanned on the 3.0Tesla MRI scanner (Signa Hdx General Electric Healthcare, Milwaukee, WI, USA) equipped with a dedicated eight-channel head coil. We performed high resolution anatomic $\mathrm{T} 1$ weighted fast spoiled gradient echo (FSPGR) using these sequence parameters; TR $=7.6 \mathrm{~ms}, \mathrm{TE}=2.1-12 \mathrm{~ms}, \mathrm{FOV}=31 \times 31 \mathrm{~cm}$, matrix $=256 \times 256$, slice thickness $=1.2 \mathrm{~mm}$ iso-voxel, slice overlap $=0.6 \mathrm{~mm}$, and image scan time of $3 \mathrm{~min}$ and $48 \mathrm{~s}$. TE time ranged between 2.1 and $12 \mathrm{~ms}$ for the imaging acquisition to accommodate the coverage. We utilised the Singlevoxel spectroscopy (SVS) technique using a point-resolved single-voxel spectroscopy (PRESS) probe-p sequence with
Table 1 Patient demographics with glaucoma grading

\begin{tabular}{lllll}
\hline & & Healthy subjects & Mild glaucoma & Severe glaucoma \\
\hline Number of optic radiation & & 15 & 15 & 15 \\
Gender & Male & $12(80 \%)$ & $6(40 \%)$ & $9(60 \%)$ \\
& Female & $3(20 \%)$ & $9(60 \%)$ & $6(40 \%)$ \\
Age (mean \pm SD in years) & & $53.9( \pm 8.0)$ & $64.9( \pm 8.2)$ & $69.7( \pm 8.2)$ \\
Mean RNFL thickness $(\mu \mathrm{m})$ & Right eye & $84.47 \pm 10.44$ & $76.37 \pm 11.35$ & $58.50 \pm 6.97$ \\
& Left eye & $83.60 \pm 10.36$ & $76.87 \pm 12.01$ & $58.60 \pm 9.05$ \\
Visual field MD scores $(\mathrm{dB})$ & Right eye & $-0.99 \pm 0.92$ & $-3.95 \pm 1.40$ & $21.98 \pm 7.35$ \\
& Left eye & $-0.90 \pm 0.84$ & $-4.05 \pm 1.15$ & $20.17 \pm 9.46$ \\
\hline
\end{tabular}

$S D$ standard deviation, $R N F L$ retinal nerve fibre layer, $M D$ mean deviation 
echo time (TE) of $35 \mathrm{~ms}$ and repetition time (TR) of $1500 \mathrm{~ms}$. Other acquisition parameters were VOI size of $20 \times 20 \times 20 \mathrm{~mm}$, matrix of $1.0 \times 1.0$, slice thickness of $20 \mathrm{~mm}$, number of excitation $(\mathrm{NEX})=128$, and scan time of $3 \mathrm{~min}$ and $48 \mathrm{~s}$. The axial, coronal, and sagittal multi-planar 3D reformatted FSPGR images were used to position a single voxel in the optic radiations. The VOI was placed on the optic radiations of each cerebral hemisphere and standardised along the calcarine sulcus, as far back as the occipital lobe and towards the midline of the brain (Fig. 1). To mitigate shimming and water suppression effects, the VOI placement, as well as contact with the bone, subcutaneous fat, vasculature, and other tissues, was avoided [16].

The raw data (P-files) obtained from the SVS measurement were then transferred and saved into a Linux system. Postprocessing was performed using the LCModel Software Version 6.2 (C) 1992-2010 by Stephen Provencher (V6.2, LCMODEL Inc., Canada) [26], where the spectra were fitted, and the metabolites were quantified. The standard basis set provided by LCModel was used, and no spectral apodization was performed. The data are zero-filled once in LCModel. This puts all the information in the real part of the spectrum. Further zero-filling adds no information [27]. The reliability of the metabolite quantification was characterised by modelling the normal distribution of the estimated percentage standard deviations (\%SD) on a per-metabolite basis. Specifically, the $\% \mathrm{SD}$ mean and standard deviation within the control group were determined for each metabolite. A $95 \%$ confidence limit (approximately, mean $+2 \times$ standard deviation) was calculated, and all values with \%SD exceeding the respective \%SD limit were omitted from further analysis. We activated all the metabolites, lipids and macromolecules in LCModel; however, in this article, we were focusing on NAA, NAAG, Cr, PCr, GPC, PCh, Gln and Glu measurement (Fig. 2). Our concentrations were in 'institutional units', selfconsistent, but equal to $\mathrm{mM}$ per $\mathrm{Kg}$ wet tissue weight by an unknown factor.

In this study, the total choline concentration was derived from the sum of glycerophosphocholine (GPC) and phosphocholine $(\mathrm{PCh})$, with the variable represented as tCho. Total creatine concentration was derived from the sum of creatine $(\mathrm{Cr})$ and phosphocreatine $(\mathrm{PCr})$, with the variable represented as $\mathrm{tCr}$. Total glutamate-glutamine was derived from the sum of glutamate (Glu) and glutamine (Gln), with the variable represented as Glx. Total N-acetyl aspartate was derived from the sum of $\mathrm{N}$-acetyl aspartate (NAA) and Nacetylaspartylglutamate (NAAG), with the variable represented as tNAA.

\section{Statistical analysis}

Statistical analyses were performed using the SPSS Version 19.0 (SPSS Inc., Chicago, IL, USA). Multiple group comparisons for metabolite concentration and metabolite concentration ratio between glaucoma grades and healthy subjects were obtained using a one-way ANOVA test for each criterion. A $p$ value of $<0.05$ was considered statistically significant.

\section{Results}

Analyses of 45 optic radiations showed findings consistent with a good, acceptable spectroscopy curve. No significant difference in mean age was observed between the mild and severe glaucoma subjects $(p>0.05)$. A significant difference in the mean age was noted between healthy and mild glaucoma subjects and between healthy and severe glaucoma subjects $(p<0.05)$.

Mean $( \pm \mathrm{SD})$ metabolite concentration and metabolite concentration ratio to $\mathrm{tCr}$ are shown in Tables 2 and 3. We found no significant difference $(p>0.05)$ between metabolite concentration and metabolite concentration ratio in the optic radiations of healthy subjects, mild glaucoma patients, and severe glaucoma patients. Concentration ratios of $\mathrm{tNAA} / \mathrm{tCr}$, tCho/ $\mathrm{tCr}$, and $\mathrm{Glx} / \mathrm{tCr}$ for the healthy subjects and the mild and severe glaucoma groups regarding optic radiation were illustrated in a boxplot (Fig. 3).
Fig. 1 VOI placement in the optic radiations using FSPGR sequence $3 \mathrm{D}$ reconstruction in the (a) sagittal, (b) axial, and (c) coronal views. Arrows indicate the VOI placement

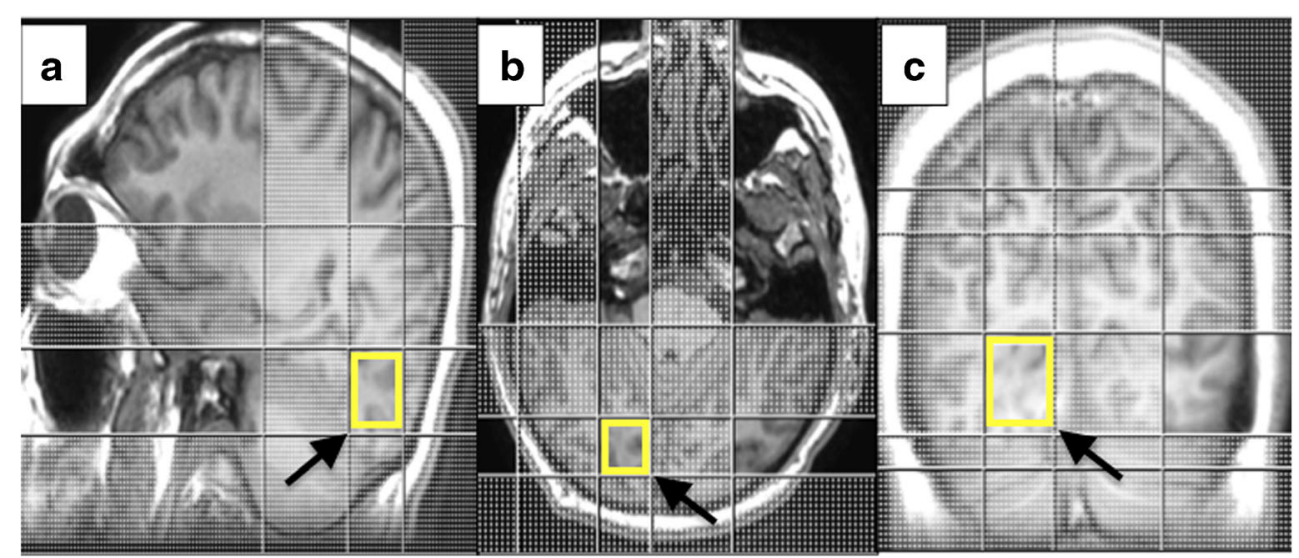




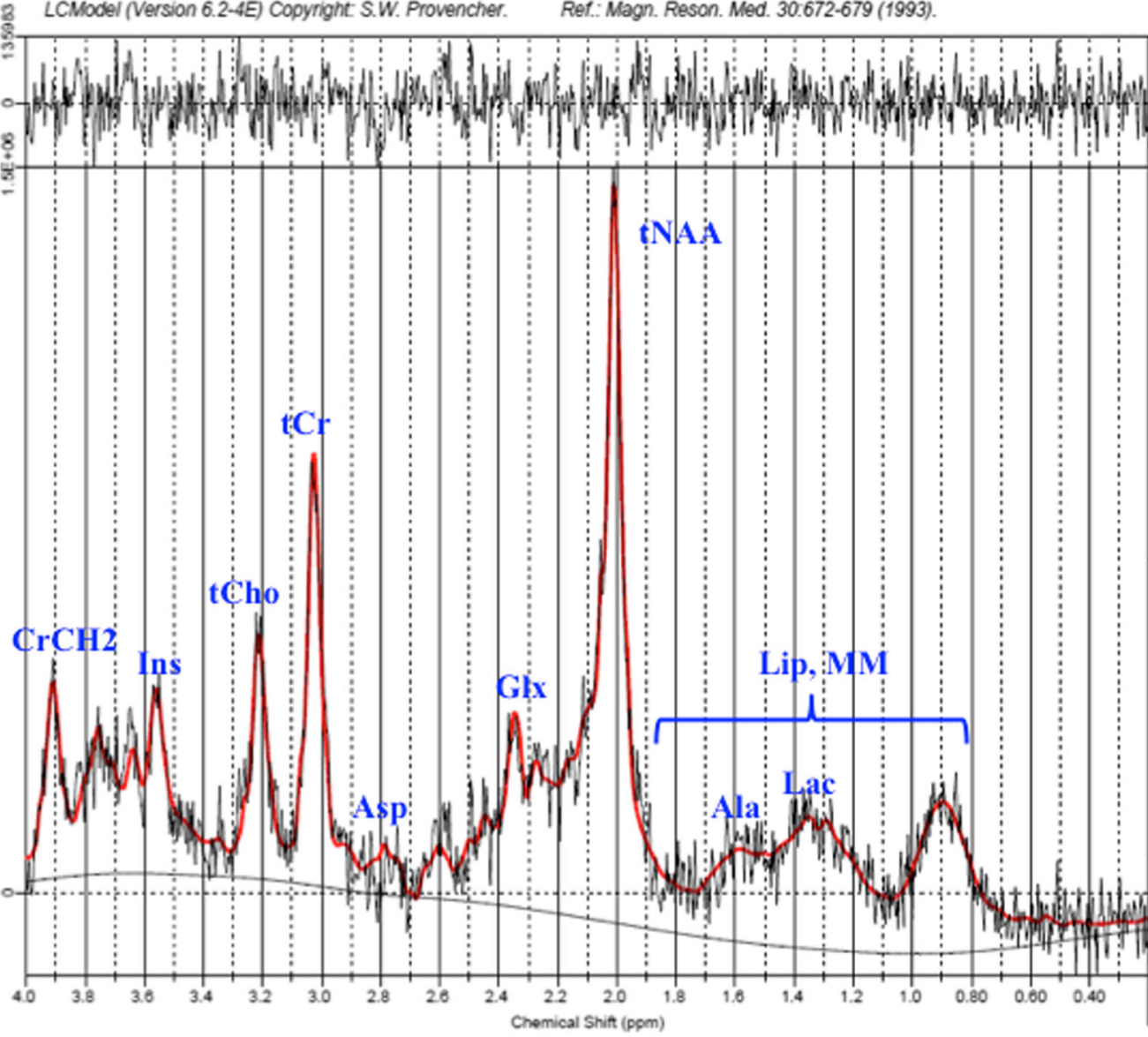

Fig. 2 Example of a good, acceptable spectroscopy spectrum from an optic radiation of a healthy subject

We also performed analysis of our data based on CramerRao lower bound (CRLB) with \%SD of less than $20 \%$ as recommended by previous studies using the LCModel [28-31]. We found significant difference in tNAA between

COne $4 \mathrm{SD} / \mathrm{Cr}+\mathrm{PCr}$ Metabolite

678-02 $8393 \quad 2.4 \mathrm{~B}-02$ Ala

$0.19816517 .6 \mathrm{E}-02$ Aap

$1.361 \quad 294 \quad 0.525 \mathrm{cr}$

$\begin{array}{llll}1.234 & 223 & 0.475 & \mathrm{PCr}\end{array}$

$\begin{array}{lll}0.496 \quad 568 & 0.107 \text { cass }\end{array}$

$\begin{array}{lll}0.472 & 543 & 0.182 \mathrm{Glc}\end{array}$

1.7692780 .682 aln

$4.387 \quad 87 \quad 2.691 \mathrm{Glu}$

$0.557 \quad 69 \quad 0.215 \mathrm{GPC}$

$0.0009998 \quad 0.000 \mathrm{DCh}$

$\begin{array}{lll}2.146 \quad 79 & 0.827 \text { Ins }\end{array}$

$0.385700 \quad 0.148$ Lae

$\begin{array}{lll}3.949 \quad 47 & 1.522 \mathrm{sma}\end{array}$

$0.2468319 .5 \mathrm{~B}-02 \mathrm{NAAG}$

5.99R-02 648 2.3R-02 Scy110

$0.100 \quad 1909 \quad 3.98-02$ Tay

$\begin{array}{lllll}1.015 & 158 & 0.391 & -\mathrm{CrCH} 2\end{array}$

$0.251 \quad 523 \quad 9.78-02$ Gua

$\begin{array}{lll}0.557 & 69 & 0.215 \mathrm{GPC}+\mathrm{FCh}\end{array}$

$2.595 \quad 39 \quad 1.000 \mathrm{CT}+\mathrm{PCE}$

$\begin{array}{llll}6.156 & 88 & 2.372 \text { Glu } & 01 \mathrm{n}\end{array}$

$2.159501 \quad 0.832$ Lip13a

$0.00099920 .000 \mathrm{~L} 1 \mathrm{p} 13 \mathrm{~b}$

$0.417 \quad 1022 \quad 0.161 .41009$

$3.300 \quad 159 \quad 1.272$ mos

0.1761518 6.0R-02 L1p20

$\begin{array}{llll}5.539 & 139 & 2.134 \quad \mathrm{mer} 20\end{array}$

$\begin{array}{llll}1.149 & 338 & 0.463 & 00012\end{array}$

$\begin{array}{llll}3.280 & 273 & 1.264 \mathrm{Mex}\end{array}$

$\begin{array}{lll}3.773 \quad 263 & 0.693 \quad \mathrm{xxy7}\end{array}$

$2.259 \quad 503 \quad 0.832$ Lip13a+Lip $13 b$

$6.589 \quad 169 \quad 2.539 \operatorname{man} 4+\mathrm{L} 1 \mathrm{p} 13 \mathrm{a}+\mathrm{t}$

$3.717 \quad 118 \quad 1.432$ may.L1p09

$5.715 \quad 139 \quad 2.202 \times 20 \times 20+21 p 20$

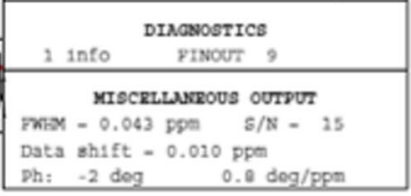

INPUT CreNogES

Table 2 Mean values of different metabolite concentrations among healthy and glaucoma subjects (based on per-metabolite basis) mild (mean \pm SD of $3.75 \pm 0.21$ ) and severe glaucoma groups (mean $\pm \mathrm{SD}$ of $3.34 \pm 0.46$ ) with $p=0.012$. No significant difference $(p>0.05)$ was found between healthy subject (mean $\pm \mathrm{SD}$ of $3.48 \pm 0.39)$ and mild glaucoma, as well as healthy

\begin{tabular}{|c|c|c|c|c|c|c|c|}
\hline \multirow[t]{2}{*}{ Metabolites } & \multicolumn{2}{|c|}{ Healthy subjects } & \multicolumn{2}{|c|}{ Mild glaucoma } & \multicolumn{2}{|c|}{ Severe glaucoma } & \multirow[t]{2}{*}{$p$ value } \\
\hline & $n$ & Mean $( \pm \mathrm{SD})$ & $n$ & Mean $( \pm \mathrm{SD})$ & $n$ & Mean $( \pm \mathrm{SD})$ & \\
\hline Creatine $(\mathrm{Cr})$ & 7 & $0.92 \pm 0.31$ & 11 & $1.03 \pm 0.25$ & 5 & $0.97 \pm 0.25$ & 0.627 \\
\hline Phosphocreatine (PCr) & 7 & $1.24 \pm 0.26$ & 11 & $1.24 \pm 0.23$ & 5 & $1.40 \pm 0.24$ & 0.479 \\
\hline Glutamine (Gln) & 7 & $1.84 \pm 0.66$ & 11 & $2.02 \pm 0.47$ & 5 & $2.14 \pm 0.48$ & 0.336 \\
\hline Glutamate (Glu) & 7 & $2.95 \pm 0.27$ & 11 & $2.95 \pm 0.42$ & 5 & $3.12 \pm 0.81$ & 0.734 \\
\hline Glycerophosphocholine (GPC) & 7 & $0.53 \pm 0.06$ & 11 & $0.54 \pm 0.09$ & 5 & $0.59 \pm 0.12$ & 0.287 \\
\hline$N$-Acetylaspartate (NAA) & 7 & $3.14 \pm 0.18$ & 11 & $3.25 \pm 0.25$ & 5 & $3.30 \pm 0.43$ & 0.210 \\
\hline $\begin{array}{l}N \text {-Acetylaspartylglutamate } \\
\text { (NAAG) }\end{array}$ & 7 & $0.42 \pm 0.16$ & 11 & $0.53 \pm 0.16$ & 5 & $0.55 \pm 0.29$ & 0.798 \\
\hline GPC + PCh (tCho) & 7 & $0.53 \pm 0.06$ & 11 & $0.54 \pm 0.09$ & 5 & $0.59 \pm 0.12$ & 0.287 \\
\hline NAA + NAAG (tNAA) & 7 & $3.57 \pm 0.26$ & 11 & $3.78 \pm 0.21$ & 5 & $3.83 \pm 0.32$ & 0.088 \\
\hline Glu + Gln (Glx) & 7 & $4.79 \pm 0.78$ & 11 & $4.97 \pm 0.59$ & 5 & $5.33 \pm 0.50$ & 0.311 \\
\hline $\mathrm{Cr}+\mathrm{PCr}(\mathrm{tCr})$ & 7 & $2.15 \pm 0.15$ & 11 & $2.28 \pm 0.24$ & 5 & $2.37 \pm 0.28$ & 0.103 \\
\hline
\end{tabular}

Data were expressed as mean $( \pm \mathrm{SD})$ and analysed using one-way ANOVA. $n$ number of subjects, $S D$ standard deviation 
Table 3 Mean concentration ratios of $\mathrm{tNAA} / \mathrm{tCr}$, $\mathrm{tCho} / \mathrm{tCr}$, and $\mathrm{Glx} / \mathrm{tCr}$ among healthy and glaucoma subjects (based on permetabolite basis)

\begin{tabular}{|c|c|c|c|c|c|c|c|}
\hline \multirow[t]{2}{*}{ Metabolite ratio } & \multicolumn{2}{|c|}{ Healthy subjects } & \multicolumn{2}{|c|}{ Mild glaucoma } & \multicolumn{2}{|c|}{ Severe glaucoma } & \multirow[t]{2}{*}{$p$ value } \\
\hline & $n$ & Mean $( \pm$ SD) & $n$ & Mean $( \pm \mathrm{SD})$ & $n$ & Mean $( \pm \mathrm{SD})$ & \\
\hline $\mathrm{tNAA} / \mathrm{tCr}$ & 15 & $1.63 \pm 0.17$ & 15 & $1.67 \pm 0.12$ & 15 & $1.55 \pm 0.16$ & 0.23 \\
\hline $\mathrm{tCho} / \mathrm{tCr}$ & 15 & $0.24 \pm 0.03$ & 15 & $0.24 \pm 0.03$ & 15 & $0.25 \pm 0.03$ & 0.80 \\
\hline $\mathrm{Glx} / \mathrm{tCr}$ & 15 & $2.20 \pm 0.30$ & 15 & $2.19 \pm 0.26$ & 15 & $2.24 \pm 0.26$ & 0.86 \\
\hline
\end{tabular}

Data were expressed as mean $( \pm \mathrm{SD})$ and analysed using one-way ANOVA. $n$ number of subjects, $S D$ standard deviation subjects and severe glaucoma groups. We have also noted that the $p$ value for Gln was 0.001 . However, statistically, the significant value was not reliable. This was due to statistical limitation to perform post Hoc tests because at least one group has fewer than two cases. Otherwise, there was no significant difference found for other metabolites, as well as between metabolite concentration ratio and $\mathrm{tCr}(p>0.05)$.

The range of metabolite concentration ratio in the optic radiation among healthy subjects was determined. The range for $\mathrm{tNAA} / \mathrm{tCr}$ was 1.3 to 1.9 , that for $\mathrm{tCho/tCr}$ was 0.2 to 0.3 , and that for $\mathrm{Glx} / \mathrm{tCr}$ was 1.8 to 2.9 .

\section{Discussion}

In our study, using CRLB analysis with \%SD of less than $20 \%$ [28-31], we found a spurious increase in the mean of tNAA in mild glaucoma compared to severe glaucoma groups, causing a significant difference in the $p$ value.
However, analysis of the data by modelling the normal distribution of the estimated percentage standard deviations (\%SD) on a per-metabolite basis, tNAA concentration was found to be non-significant across the groups. The differences in the findings were attributed to the comparison of absolute CRLB to those of the healthy subject group. The CRLB to normal metabolite levels as shown in this study was more useful as quality criteria [32]. Furthermore, bias could also be avoided, as low levels of metabolite concentrations of interest was also included in the analysis [32].

Either using CRLB with \%SD $<20 \%$ or absolute CRLB (with comparison to the healthy subject), the metabolite concentration ratios $(\mathrm{tNAA} / \mathrm{tCr}, \mathrm{tCho} / \mathrm{tCr}$ and $\mathrm{Glx} / \mathrm{tCr}$ ) were found to be nonsignificant across the glaucoma grades and healthy subjects. Metabolite concentration ratios are more sensitive regarding detecting changes, and can be more accurate than absolute concentration due to application of specific characteristics in the computer program analysis [33, 34]. Therefore, from this study, the ratio of metabolite
Fig. 3 Boxplot illustrating concentration ratios of tNAA, tCho, and Glx to $\mathrm{tCr}$ for the healthy subjects and the mild and severe glaucoma groups in terms of optic radiations (based on permetabolite basis)

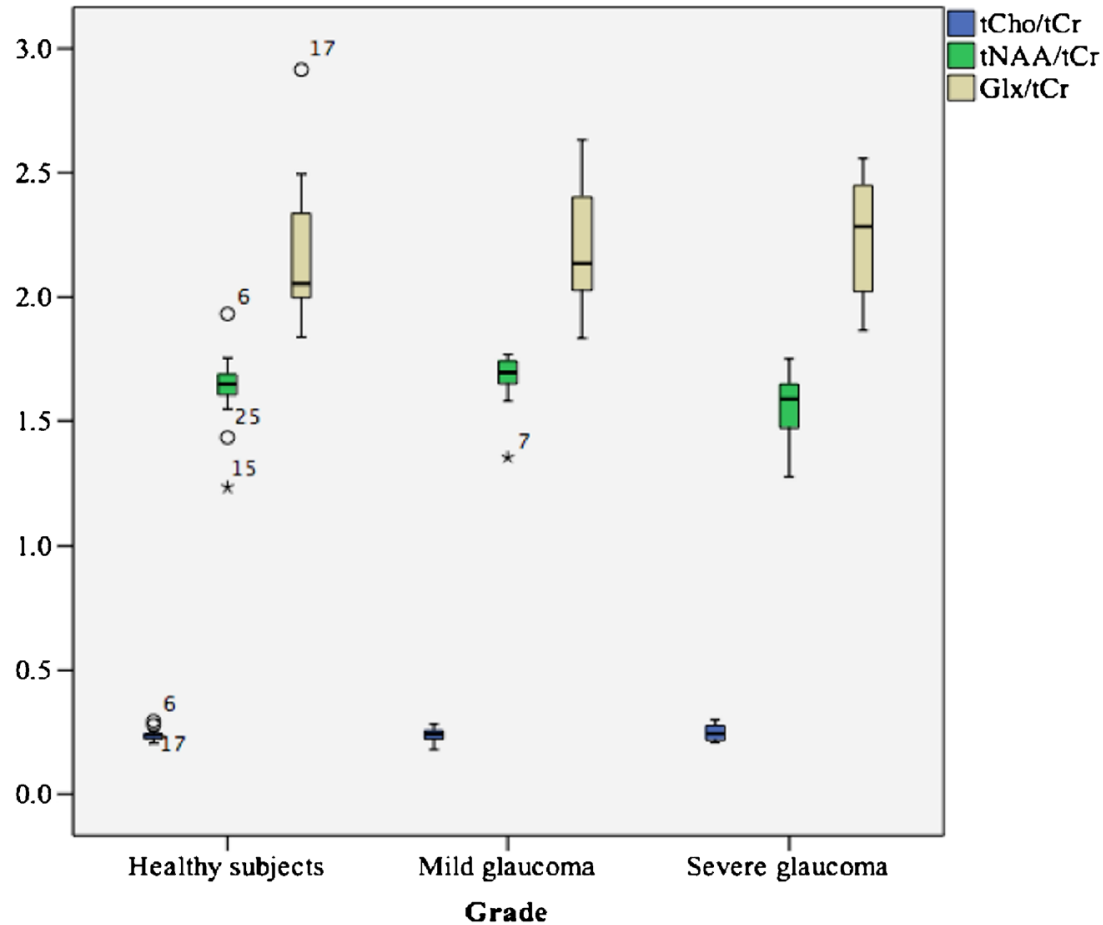


concentration to creatine was more reliable to represent the changes in optic radiations. Our results corroborated the findings of Boucard et al., who concluded that progressive retinal visual field defects do not affect absolute metabolite concentration in optic radiations, thus implying the absence of ongoing occipital degeneration attributable to slow detectable metabolite changes [16].

From this study, we also obtained the ranges of metabolite ratios in optic radiation among healthy subjects. The ranges for the $\mathrm{tNAA} / \mathrm{tCr}, \mathrm{tCho} / \mathrm{tCr}$, and $\mathrm{Glx} / \mathrm{tCr}$ ratios were 1.3 to 1.9 , 0.2 to 0.3 , and 1.8 to $2.9 \mathrm{~m}$, respectively. The differences between the ranges obtained in this study from those obtained in other studies on healthy subjects may be attributed to the site of measurement. This is supported by previous studies performed by Ludovico et al., Pouwels et al., and Grachev et al., who all found various significant metabolite concentrations across regional normal brain structures, owing to the chemical heterogeneity of the human brain [35-37].

A study conducted by Yan Zhang et al. revealed a significant reduction in NAA/Cr and $\mathrm{Cho} / \mathrm{Cr}$ in primary glaucoma using multi-voxel 1H-MRS [18]. The reasons for the differences between our study and this study may be attributed to the differences in the applied technique, with the previous work using ratios instead of absolute measurements and multi-voxel instead of SVS, and in the site of measurement. Multi-voxel spectroscopy obtains multiple single-voxel spectra, which can reduce the partial volume effect while acquiring a higher signal because of its large view, which can reflect the spatial distribution of metabolites. However, the capability of multi-voxel spectroscopy for multi-dimensional data acquisition is more appropriate for the measurement of diffuse, inhomogeneous, and large areas of pathological changes in the brain, such as a tumour. In this research, we considered a specific region, which was the optic radiation, such that we did not require the use of multi-voxel. Furthermore, a few disadvantages, such as insufficient water suppression, poor shimming, and voxel bleeding, contribute to inability to acquire high-quality spectra in multi-voxel [38].

Another study by Doganay et al. [17] differs from our study with respect to the site of measurement. There were indeed differences in both technique and patient selection. Their study had 13 normal patients, and two mild glaucoma, 18 moderate and nine severe glaucoma patients according to Hodapp Anderson criteria. Ours had 15 normal, 15 mild and 15 severe. Hence it is not entirely comparable as they had more numbers of 'diseased' patients. More importantly, in terms of technique as well, they looked at concentrations in vitreous (within the eyeball) and lateral geniculate body (LGB). Our study looked at more distal sites in the optic radiations. They found significant positive differences at level of vitreous for lactate and Glx/Cr, but not in LGB. This implies that the primary site of metabolite differences lies more proximal in location, nearer to optic nerve rather than further down the optic tracts. They also did not specify who of these 11 patients had significant differences, what grade of glaucoma severity they had and whether it correlated with disease severity or not. Therefore, future research area should concentrate on metabolite differences in areas more proximal, i.e., vitreous and optic nerve, since our results demonstrated no significant change in the optic radiations despite clinical evidence of severe glaucoma.

Chan et al. carried out an in vivo spectroscopy study of glaucoma in rats where ocular hypertension was unilaterally induced in the right eye of five Sprague-Dawley rats [19]. Single-voxel 1H-MRS was performed after 6 weeks of induction at the left visual cortex. The left eye and right visual cortex were considered as internal control. They found significant $\mathrm{Cho} / \mathrm{Cr}$ reduction in the left visual cortex compared with the right. No significant difference was observed in the other metabolites, including NAA and Glx. Therefore, they concluded that glaucoma alters choline metabolism in the visual cortex [19]. This finding needs to be confirmed in human studies.

A major limitation of our study is the small size of optic radiation compared with the size of the spectroscopic volume of interest. Our area of measurement may be also too distal to the area of primary degeneration, which occurs at the level of the optic nerve head in glaucoma. Another study that focuses on the metabolite concentrations within the optic nerves or optic tracts may yield significant results. The difference in mean age between the healthy and the mild glaucoma group, as well as between the healthy and severe glaucoma groups, could also be a limiting factor in our study. Patients with mild and severe glaucoma are significantly older than those in the control group. This is a limitation of the study, given that metabolite concentrations were reported to change slightly with age $[39,40]$. Nevertheless, this condition is not likely to affect the results of this study. Also, we did not perform test-retest analysis to obtain real reproducibility findings in this study.

In conclusion, the result of this cross-sectional study showed that no significant metabolite changes in the optic radiation were associated with glaucoma and its severity, which could be measured by single-voxel 1H-MRS.

Acknowledgments The scientific guarantor of this publication is Professor Dr Norlisah Ramli. The authors of this manuscript declare no relationships with any companies, whose products or services may be related to the subject matter of the article. This study has received funding by the University of Malaya Institutional Research Grants (GRANT NOS: RG390-11HTM, J-20518-73808, P0083/2012A and RP008C$13 \mathrm{HTM}$ ), and the Ministry of Higher Education, Government of Malaysia [GRANT NO: 600-RMI/RAGS 5/3 (40/2014)]. No complex statistical methods were necessary for this paper. Institutional Review Board approval was obtained. The approval body was the University of Malaya Research Ethics Committee (UMREC). Written informed consent was obtained from all subjects (patients) in this study. 
Some study subjects or cohorts have been previously reported in:

i) Sidek S, Ramli N, Rahmat K, Ramli NM, Abdulrahman F, Tan LK. Glaucoma severity affects diffusion tensor imaging (DTI) parameters of the optic nerve and optic radiation. European journal of radiology. 2014 Aug. 83(8):1437-41. PubMed PMID: 24908588.

ii) Ramli NM, Sidek S, Rahman FA, Peyman M, Zahari M, Rahmat K, et al. Novel use of $3 \mathrm{~T}$ MRI in assessment of optic nerve volume in glaucoma. Graefe's archive for clinical and experimental ophthalmology $=$ Albrecht von Graefes Archiv fur klinische und experimentelle Ophthalmologie. 2014 Jun; 252(6):995-1000. PubMed PMID: 24770532.

Methodology: cross sectional study, performed at one institution.

Open Access This article is distributed under the terms of the Creative Commons Attribution-NonCommercial 4.0 International License (http:// creativecommons.org/licenses/by-nc/4.0/), which permits any noncommercial use, distribution, and reproduction in any medium, provided you give appropriate credit to the original author(s) and the source, provide a link to the Creative Commons license, and indicate if changes were made.

\section{References}

1. Quigley HA, Broman AT (2006) The number of people with glaucoma worldwide in 2010 and 2020. Br J Ophthalmol 90(3):262267

2. Agarwal R, Gupta SK, Agarwal P, Saxena R, Agrawal SS (2009) Current concepts in the pathophysiology of glaucoma. Indian $\mathrm{J}$ Ophthalmol 57(4):257-266

3. Garaci FG, Bolacchi F, Cerulli A et al (2009) Optic nerve and optic radiation neurodegeneration in patients with glaucoma: in vivo analysis with 3-T diffusion-tensor MR imaging. Radiology 252(2):496-501

4. Gupta N, Ang LC, Noel de Tilly L, Bidaisee L, Yucel YH (2006) Human glaucoma and neural degeneration in intracranial optic nerve, lateral geniculate nucleus, and visual cortex. Br J Ophthalmol 90(6):674-678

5. Chen Z, Lin F, Wang J et al (2013) Diffusion tensor magnetic resonance imaging reveals visual pathway damage that correlates with clinical severity in glaucoma. Clin Experiment Ophthalmol 41(1):43-49

6. Ramli NM, Sidek S, Rahman FA, et al. (2014) Novel use of 3T MRI in assessment of optic nerve volume in glaucoma. Graefes Arch Clin Exp Ophthalmol 252(6):995-1000

7. Sidek S, Ramli N, Rahmat K, Ramli NM, Abdulrahman F, Tan LK (2014) Glaucoma severity affects diffusion tensor imaging (DTI) parameters of the optic nerve and optic radiation. Eur J Radiol 83(8):1437-1441

8. Hernowo AT, Boucard CC, Jansonius NM, Hooymans JM, Cornelissen FW (2011) Automated morphometry of the visual pathway in primary open-angle glaucoma. Invest Ophthalmol Vis Sci 52(5):2758-2766

9. Bogorodzki P, Piatkowska-Janko E, Szaflik J et al (2014) Mapping cortical thickness of the patients with unilateral end-stage open angle glaucoma on planar cerebral cortex maps. PLoS One 9(4), e93682

10. Yu L, Xie B, Yin X et al (2013) Reduced cortical thickness in primary open-angle glaucoma and its relationship to the retinal nerve fiber layer thickness. PLoS One 8(9), e73208

11. Zikou AK, Kitsos G, Tzarouchi LC et al (2012) Voxel-based morphometry and diffusion tensor imaging of the optic pathway in primary open-angle glaucoma: a preliminary study. AJNR Am J Neuroradiol 33(1):128-134
12. Lu P, Shi L, Du H et al (2013) Reduced white matter integrity in primary open-angle glaucoma: a DTI study using tract-based spatial statistics. J Neuroradiol 40(2):89-93

13. Williams AL, Lackey J, Wizov SS et al (2013) Evidence for widespread structural brain changes in glaucoma: a preliminary voxelbased MRI study. Invest Ophthalmol Vis Sci 54(8):5880-5887

14. Li C, Cai P, Shi L et al (2012) Voxel-based morphometry of the visual-related cortex in primary open angle glaucoma. Curr Eye Res 37(9):794-802

15. Chen WW, Wang N, Cai S et al (2013) Structural brain abnormalities in patients with primary open-angle glaucoma: a study with $3 \mathrm{~T}$ MR imaging. Invest Ophthalmol Vis Sci 54(1):545-554

16. Boucard CC, Hoogduin JM, van der Grond J, Cornelissen FW (2007) Occipital proton magnetic resonance spectroscopy $(1 \mathrm{H}-$ MRS) reveals normal metabolite concentrations in retinal visual field defects. PLoS One 2(2), e222

17. Doganay S, Cankaya C, Alkan A (2012) Evaluation of corpus geniculatum laterale and vitreous fluid by magnetic resonance spectroscopy in patients with glaucoma; a preliminary study. Eye (Lond) 26(8):1044-1051

18. Zhang Y, Chen X, Wen G, Wu G, Zhang X (2013) Proton magnetic resonance spectroscopy ((1)H-MRS) reveals geniculocalcarine and striate area degeneration in primary glaucoma. PLoS One 8(8), e73197

19. Chan KC, So KF, Wu EX (2009) Proton magnetic resonance spectroscopy revealed choline reduction in the visual cortex in an experimental model of chronic glaucoma. Exp Eye Res 88(1):65-70

20. Castillo M, Kwock L, Mukherji SK (1996) Clinical applications of proton MR spectroscopy. AJNR Am J Neuroradiol 17(1):1-15

21. Gujar SK, Maheshwari S, Bjorkman-Burtscher I, Sundgren PC (2005) Magnetic resonance spectroscopy. J Neuroophthalmol 25(3):217-226

22. Soares DP, Law M (2009) Magnetic resonance spectroscopy of the brain: review of metabolites and clinical applications. Clin Radiol 64(1): 12-21

23. Ross B, Michaelis T (1994) Clinical applications of magnetic resonance spectroscopy. Magn Reson Q 10(4):191-247

24. Ross B, Bluml S (2001) Magnetic resonance spectroscopy of the human brain. Anat Rec 265(2):54-84

25. Hodapp EPR, Anderson DR (1993) Clinical decisions in glaucoma, vol 1. Mosby, St Louis

26. SW P (2011) LcModel and LcMgui user's manual. Available via http://s-provencher. com/pages/lcmodel.shtml. Accessed 26 January 2015

27. Bartholdi E, Ernst RR (1973) Fourier spectroscopy and the causality principle. J Magn Reson 11:9-19

28. Cavassila S, Deval S, Huegen C, van Ormondt D, GraveronDemilly D (2000) Cramer-Rao bound expressions for parametric estimation of overlapping peaks: influence of prior knowledge. J Magn Reson 143:311-320

29. Cavassila S, Deval S, Huegen C, van Ormondt D, GraveronDemilly D (2001) Cramer-Rao bounds: an evaluation tool for quantitation. NMR Biomed 14(4):278-283

30. Provencher SW (1993) Estimation of metabolite concentrations from localized in vivo proton NMR spectra. Magn Reson Med 30(6):672-679

31. Provencher SW (2001) Automatic quantitation of localized in vivo 1H spectra with LCModel. NMR Biomed 14(4):260-264

32. Kreis R (2015) The trouble with quality filtering based on relative Cramer-Rao Lower Bounds. Magn Reson Med 75:15-18

33. Kanowski M, Kaufmann J, Braun J, Bernar- ding J, Tempelmann C (2004) Quantitation of simulated short echo time 1H human brain spectra by LCModel and AMARES. Magn Reson Med 51:904 912 
34. Jansen JF, Backes WH, Nicolay K, Kooi ME (2006) 1H MR spectroscopy of the brain: absolute quantification of metabolites. Radiology 240(2):318-332

35. Minati L, Aquino D, Bruzzone MG (2010) Quantitation of normal metabolite concentrations in six brain regions by in-vivo $1 \mathrm{H}-\mathrm{MR}$ spectroscopy. J Med Phys 35(3):154-163

36. Grachev ID, Apkarian AV (2000) Chemical heterogeneity of the living human brain: a proton MR spectroscopy study on the effects of sex, age, and brain region. Neuroimage 11(5 Pt 1):554-563

37. Pouwels PJ, Frahm J (1998) Regional metabolite concentrations in human brain as determined by quantitative localized proton MRS. Magn Reson Med 39(1):53-60
38. Yang ZX, Huo SS, Cheng XF et al (2012) Quantitative multi-voxel proton MR spectroscopy study of brain metabolites in patients with amnestic mild cognitive impairment: a pilot study. Neuroradiology 54(5):451-458

39. Harris JL, Yeh HW, Swerdlow RH, Choi IY, Lee P, Brooks WM (2014) High-field proton magnetic resonance spectroscopy reveals metabolic effects of normal brain aging. Neurobiol Aging 35(7): 1686-1694

40. Haga KK, Khor YP, Farrall A, Wardlaw JM (2009) A systematic review of brain metabolite changes, measured with $1 \mathrm{H}$ magnetic resonance spectroscopy, in healthy aging. Neurobiol Aging 30(3): $353-363$ 